\title{
High number of chlamydia trachomatis plasmid copy number in clinical urogenital samples: diagnostic impact
}

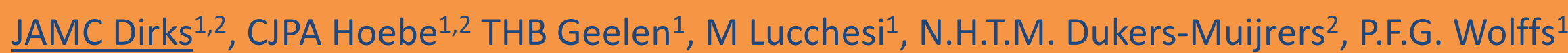

1 Department of Medical Microbiology, School of Public Health and Primary Care, Maastricht University Medical Center, Maastricht, The Netherland

2 Department of Sexual Health, Infectious Diseases, and Environmental Health, South Limburg Public Health Service, Geleen, The Netherlands

Contact: Anne.Dirks@mumc.nl

\section{Introduction}

The Chlamydia trachomatis (CT) cryptic plasmid, an extra-chromosomal genetic elements capable of self-replication, confers a survival benefit by regulating chromosomal gene expression and virulence. Plasmidless strains have been reported, but are rare and exhibit a diminished virulence. The number of CT plasmids in laboratory strains is stable and ranges from 1-10 plasmid per bacterium in vitro. It has been demonstrated that the plasmid copy number is more variable in clinical trachoma samples, with 1-18 plasmid per bacterium [8]. Little is known about plasmid variation in other samples, which is why we assessed plasmid copy numbers in urogenital samples.

\section{Methods}

- 1331 patients from the STI-clinic in South Limburg, the Netherlands and the general population (participating in a mail-based CT screening = population-based screening (PBS) ), of which 962 generated valid results

- First-void urines for men and self-collected vaginal swabs for women

- Urine transported without a stabilising buffer

- Vaginal swabs transported and stored in a stabilising buffer

- Chromosomal CT copies (OmpA-gene) and plasmids were retrospectively quantified

- Plasmid genome ratio $(\mathrm{PGr})=$ chromosomal copies $/ \mathrm{ml}$ plasmid copies/ml

- Questionnaires provided information about age, gender, ethnicity, previous STI's and symptoms

- Statisitical analyses were performed using non-parametric independent-sample tests were used

\section{Results}

- Majority (77\%) contained PGr 1 - 35: 34\% PGr 1-10, 52.0\% PGr 1-15 and 62\% PGR 1-20.

- Different PGr in different sample types:

- $P G r$ in vaginal swabs $(n=707)$ : median 13 , range $0.002-15368$

- Pgr in urines ( $n=239)$ : median 26, range 2-29516

- Different PGr in different cohorts:

- PGr in PBS ( $n=557):$ median 15 [0-1524] and $\widehat{o}$ median 39 [4-1001]

- PGr in STI-clinic ( $\mathrm{n=389)}$ : $q$ median 10 [0-15386] and ô median 22 [2-29516]

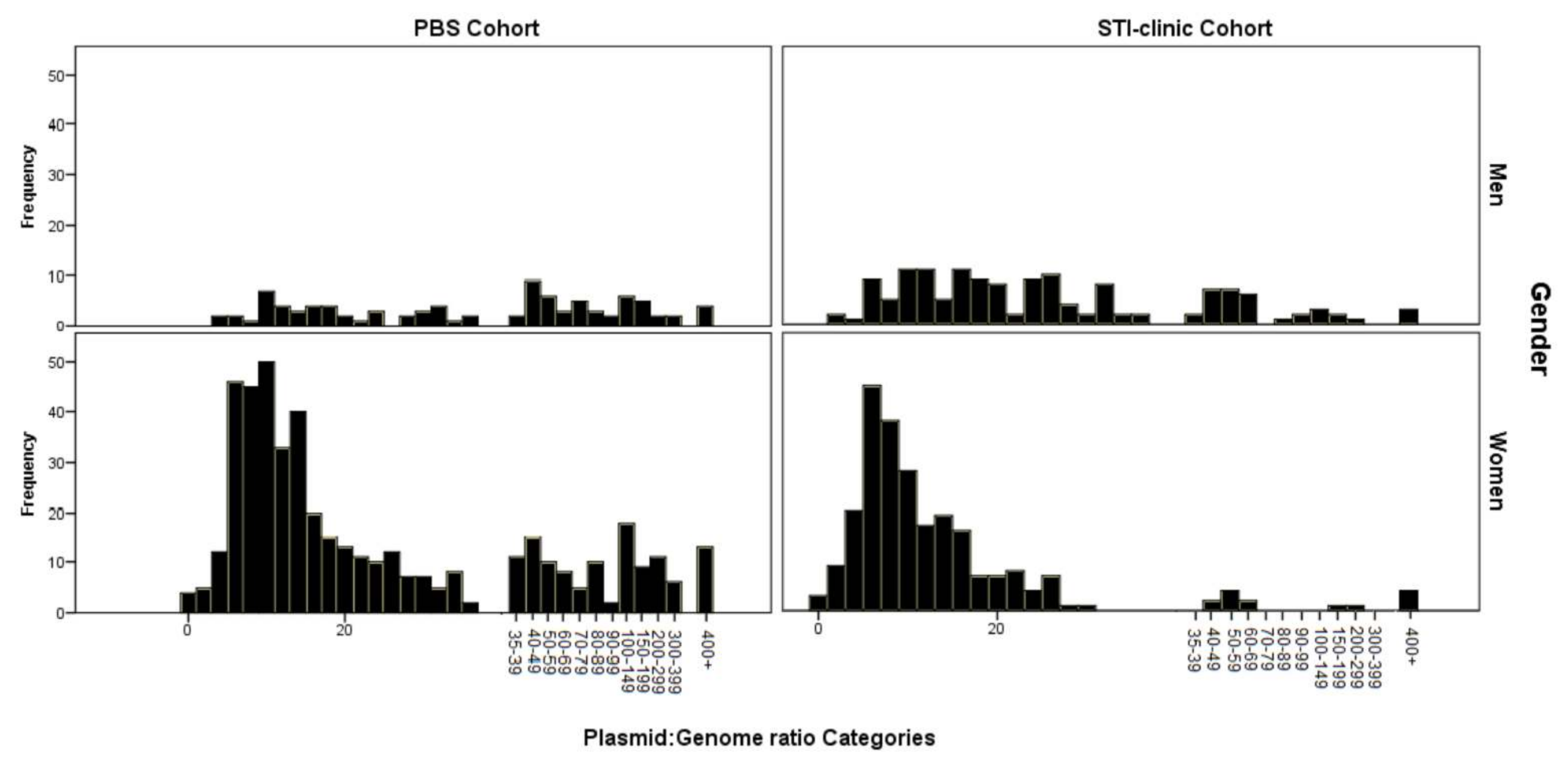

\section{Conclusion}

Higher variability of the PGr was demonstrated in self-collected urogenital samples from patients visiting the STI-clinic or participating in a Dutch population-based-screening than was previously described $(1,2)$. This may be due to natural variation and/or differences in degradation of the small plasmid and larger bacterial chromosome within a sample. These results support the use of the CT plasmid to increase the sensitivity of screening assays. Yet, of the five FDA approved and commercially available NAATS (Roche Diagnostics, Becton Dickinson and Company, Hologic/Gen-Probe Inc., Abbott Laboratories, and Cepheid), one detects only a chromosomal target, two only plasmid targets, one detects rRNA and only one detects both. 